\title{
Activation of autophagy ameliorates age-related neurogenesis decline and neurodysfunction in adult mice
}

na yang ( 18234046504@163.com)

Department of anatomy https://orcid.org/0000-0003-0610-0902

Xue-qin Liu

Shanxi Medical University

Xiao-jie Niu

Shanxi Medical University

Xiao-qiang Wang

Shanxi Medical University

Rong Jiang

Shanxi Medical University

Na Yuan

Soochow University School of Medicine

Jian-rong Wang

Soochow University School of Medicine

Cheng-wu Zhang

Nanjing Tech University

Kah-Leong Lim

Lee Kong Chian School of Medicine

Li Lu

Shanxi Medical University

\section{Research Article}

Keywords: autophagy, neural progenitor cell, adult neurogenesis, rapamycin, mTOR

Posted Date: April 8th, 2021

DOl: https://doi.org/10.21203/rs.3.rs-359844/v1

License: (c) (i) This work is licensed under a Creative Commons Attribution 4.0 International License.

Read Full License 
Version of Record: A version of this preprint was published at Stem Cell Reviews and Reports on September 21st, 2021. See the published version at https://doi.org/10.1007/s12015-021-10265-0. 


\section{Abstract}

Adult neurogenesis is the ongoing generation of functional new neurons from neural progenitor cells (NPCs) in the mammalian brain. However, this process declines with aging, which is implicated in the recession of brain function and neurodegeneration. Understanding the mechanism of adult neurogenesis and stimulating it will benefit the mitigation of neurodegenerative diseases. Autophagy, a highly conserved process of cellular degradation, is essential for maintaining cellular homeostasis and normal function. Whether and how autophagy affects adult neurogenesis remains poorly understood. In present study, we revealed a close connection between impaired autophagy and adult neurogenetic decline. Expression of autophagy-related genes and autophagic activity were significantly declined in the middleaged subventricular/subgranular zone (SVZ/SGZ) homogenates and cultured NPCs, and inhibiting autophagy by siRNA interference resulted in impaired pluripotency of NPCs. Conversely, stimulating autophagy by rapamycin not only revitalized the viability and pluripotency of middle-aged NPCs, but also facilitated the neurogenesis in middle-aged SVZ/SGZ. More importantly, autophagic activation by rapamycin also ameliorated the olfactory sensitivity and cognitional capacities in middle-aged mice. Taken together, our results reveal that compromised autophagy is involved in the decline of adult neurogenesis, which could be reversed by autophagy activation. It also shed light on the regulation of adult neurogenesis and paves the way for developing therapeutic strategy for aging and neurodegenerative diseases.

\section{Introduction}

Neurogenesis is the process of formation of neurons de novo by neural progenitor cells (NPCs). In adult rodents, neurogenesis mainly occurs in two neurogenic niches: the subventricular zone (SVZ) located along the walls of the lateral ventricles and the subgranular zone (SGZ) of the dentate gyrus (DG) in the hippocampus [1]. Neurons born in the adult rodent SVZ migrate a long distance along the rostral migratory stream (RSM) to the olfactory neuroepithelium to initiate the neurogenesis of the olfactory bulb, while neurons born in the adult SGZ migrate a comparatively shorter distance to the granule cell layer of the hippocampus, which play a pivotal role in learning and memory $[2,3]$. Adult neurogenesis in human brain has been supported by immunohistochemical analysis and non-invasive neuroimaging approaches [3-5]. However, adult neurogenesis undergoes a decline with aging. A sharp drop occurs even in as early as juvenile development of human (7-13 years old), which is attributed to the loss of NPCs number and activity $[5,6]$. In accordance with this, prior work by our group and others have revealed that adult neurogenesis exhibits a dramatic decline in mice from 6-9 months of age and becomes lower or undetectable with further aging [7-9]. Therefore, understanding of the mechanism of adult neurogenesis and feasibly controlling it would help mitigate brain aging and neurodegenerative diseases.

Autophagy is an essential cellular homeostasis process involved in the degradation of damaged organelles and noxious proteins. Autophagic dysfunction has been implicated in age-related diseases, including neurodegenerative disorders, such as Alzheimer's disease (AD), Parkinson's disease (PD), and Huntington's disease (HD). For instance, increasing evidences have shown that inefficient autophagy 
leads to elevated a-synuclein (a-Syn) aggregation in brain and significant dopaminergic neurons loss in the substantia nigra pars compacta in PD [10-12]. Autophagy is regulated by a series of autophagyrelated genes (Atgs) acting sequentially. The deletion of Atgs (including Ambra 1, Beclin1, Fip200 and Atg5) in mice results in blocked autophagic flux and attenuated LC3-囚, an autophagic marker, which is accompanied by a progressive loss of NSCs/NPCs and a significant reduction in newborn neurons in the postnatal or early adult SVZ and hippocampus [13-15]. In addition, lacking of Atg7 expression leads to impaired autophagic activity, accompanied by the accumulation of cytoplasmic inclusion bodies and aSyn protein in neurons or massive neuronal loss that contribute to PD onset $[16,17]$. Interestingly, upregulated $A \operatorname{tg} 7$ and associated autophagic flux have been observed during the differentiation of mouse embryonic olfactory bulb stem cells (eOBSCs) [18]. Genetic suppression of Atg7leads to deferral embryogenesis, premature death, massive neuronal loss and progressive neurodegeneration phenotypes $[16,19]$.

Currently, studies on the functional effects of autophagy on neurogenesis were mainly focused on brain development stage and the early postnatal period, but little is known about its function in adult neurogenesis. Rapamycin is a well-established autophagy inducer, which can trigger autophagy initiation by selective inhibition of mTOR [20]. However, conflicting reports exist concerning the effectiveness of rapamycin on neurogenesis. Singh et a/ have reported that rapamycin reverses the decline of neuronal makers levels in aged rats possibly via reducing the aging-induced oxidative stress, apoptotic cell death, and neurodegeneration makers [21]. However, several studies have revealed that intraperitoneal injection of rapamycin leads to a decrease in progenitor stem cell pool and eliminates NSCs proliferiation in early adult hippocampus [7,22] or in injured brain [23]. Hence clarifying how autophagy affects adult neurogenesis is needed.

In present study, we explored whether autophagy impact on the decline of adult neurogenesis, and whether pharmacological enhancement of autophagy by rapamyin affects adult neurogenesis and neurological function in both SVZ and SGZ of hippocampus. Our study is aimed at providing clues for activating adult endogenous neurogenesis and delaying age-related brain dysfunction.

\section{Materials And Methods}

\section{Animals}

All experimental procedures were executed in line with the institutional guidelines approved by the Animal Research Ethics Committee at the Shanxi Medical University. C57BL/6 mice were purchased from the Animal Center of Shanxi Medical University. GFP-LC3 mice (\#BRC00806) were provided by J. Wang (Soochow University School of Medicine). All mice were kept under a 12-hr light/dark cycle, with free access to food and water, and temperature at $22^{\circ} \mathrm{C}$. In this study, neonatal mice were defined as postnatal $24 \mathrm{~h}$, young mice as 2-3 months, and middle-aged mice as 7-8 months.

\section{NPCs culture and differentiation}


Primary NPCs were isolated by dissecting the SVZ of neonatal mice or middle-aged mice following the established protocols [8]. In brief, cells were cultured to form neurospheres in proliferation medium consisting of DMEM-F12 medium supplemented with B27 (2\%; Invitrogen), basic fibroblast growth factor (bFGF, 20ng/mL; PeroTech, Rocky Hill, NJ), and epidermal growth factor (EGF, 20 ng/mL; PeroTech).

For differentiation, neurospheres were collected and digested to prepare a single cell suspension by Accutase. Then NPCs were seeded on the poly-L-ornithine $(50 \mu \mathrm{g} / \mathrm{mL}$, Sigma-Adrich, St Louis, MO) and laminin $(20 \mu \mathrm{g} / \mathrm{mL}$, Sigma-Aldrich) pre-coated coverslips. Following an overnight incubation with proliferation medium, cells were replenished with the differentiation medium containing $0.5 \%$ fetal bovine serum and 1\% B27 supplement for 5-day differentiation, followed by immunophenotypical identification of the neuronal marker Tuj1, as described below.

\section{RNA interference}

The siRNAs specifically targeting Atg7 ( 5'-GCAUCAUCUUUGAAGUGAATT-3' ) and Atg7 ( 5'CAGCCUGGCAUUUGAUAAATT-3' ) were synthesized by Genepharma Corp ( Shanghai, China ). Both siAtg7s were co-transfected into neonatal NPCs suspension via LONZA 4D-Nucleofector System (Lonza, Germany) according to the manufacturer's instructions. Gene knockdown efficiency was confirmed by qPCR and Western blotting 48- and 72-hour after incubation of siRNAs.

\section{Rapamycin treatment}

Rapamycin (Sigma) was dissolved in DMSO at a concentration of $10 \mathrm{mg} / \mathrm{ml}$ and further diluted in $1 \times$ PBS with 5\% Tween-80 and 5\% PEG-400 for application. In vitro, rapamycin was added to the middle-aged NPCs suspension at concentrations of $10 \mathrm{nM}, 20 \mathrm{nM}$ and $50 \mathrm{nM}$. In vivo, mice were intraperitoneally injected with $4 \mathrm{mg} / \mathrm{kg}$ rapamycin every other day for 4 weeks. Upon completion of rapamycin treatments, cells and mice were prepared for the following tests.

\section{Neurosphere assay}

To detect the neurosphere formation, the cultures with siRNAs transfection and rapamycin administration were observed and captured every day under a phase contrast microscope (Model CKX41, Olympus, Japan). Neurospheres were formed within 5 to 7 days, and the number and the diameter of neurospheres, with diameters larger than $30 \mu \mathrm{m}$, were measured using the Image $\mathrm{J}$ software ( $\mathrm{NIH}$, Bethesda, MD).

\section{Senescence-associated $\beta$-galactosidase staining}

The senescent cells in NPCs subjected to siRNA and rapamycin were evaluated with SA- $\beta$-gal Staining Kit (Beyotime) as suggested in the instruction. The SA- $\beta$-gal positive cells were stained into blue and counted in a double-blind manner. In each sample, 10 different non-overlapping fields under 20 -fold magnification were randomly selected, and the SA- $\beta$-gal positive ratio was calculated. 
Total RNA was extracted from NPCs isolated from neonatal, young and middle-aged mice using TRIzol reagent (Invitrogen), and was reverse-transcribed into cDNA utilizing the PrimeScriptTM RT reagent kit (Takara, RR047A, Japan) following the instruction. Gene expression levels of Atg3, Atg5, Atg7 and Beclin1 were quantified using the TB Green ${ }^{\circledR}$ Premix $\operatorname{Ex~Taq~}^{\text {TM }}$ GC (Takara, RR071A, Japan) on the StepOne Plus Real-Time PCR System (Applied Biosystems Inc., Foster City, CA). In a $20 \mu \mathrm{l}$ reaction mixture, the amount of cDNA template added was $100 \mathrm{ng}$, and the final concentration of primer was $0.2 \mu \mathrm{M}$. The mRNA levels were quantitatively analyzed by $2^{-\Delta \Delta C t}$ method and normalized with GAPDH. Sequences of primers utilized in the assays were listed in Table 1.

\section{Western blot}

Total proteins were extracted from tissues and cells using RIPA lysis buffffer supplemented with the protease inhibitor cocktail ( Thermo Fischer, Pittsburgh, PA ). Protein concentrations were determined by a BCA Protein Assay kit (Beyotime). Equal amount of proteins were separated by SDS-PAGE, and electrotransferred into PVDF membranes (Millipore, Billerica, MA, USA). After blocking with $5 \%$ skim milk in TBST ( TBS containing $0.1 \%$ Tween-20 ) for $1 \mathrm{hr}$ at room temperature, the membranes were incubated with the primary antibodies overnight at $4 \circ \mathrm{C}$. On the next day, membranes were thoroughly rinsed in the TBST for three times, followed by incubation with goat anti-rabbit IgG or goat anti-mouse IgG secondary antibodies for $2 \mathrm{hrs}$ at room temperature. After rinsing in the TBST, bands were then visualized through an enhanced ECL detection kit (GE Healthcare Life Science, Pittsburgh, PA), and documented on films. Intensities of bands were quantified using Image $\mathrm{J}$ software, and $\beta$-actin was used as a housekeeping control. Antibodies utilized here were listed in Table 2.

\section{BrdU incorporation}

In vitro, NPCs with siRNAs transfection and rapamycin administration were seeded on the pre-coated coverslips and kept in the proliferation medium overnight. $\mathrm{BrdU}(10 \mu \mathrm{M})$ was added during the last 7 hours of culture. Then, BrdU immunofluorescence detection was performed as detailed in following methods.

In vivo, middle-aged mice were intraperitoneally injected with BrdU (100 mg/kg of body weight) daily for seven consecutive days, starting from the 8-th day during rapamycin administration. Upon finishing the 28-day rapamycin injection, mice were sacrificed $24 \mathrm{hrs}$ later. For the detection of new neurons generated from NPCs in the olfactory bulb, NeuN/BrdU double staining was executed as described below.

\section{Immunofluorescence staining}

For immunocytochemistry analyses, cells were fixed with $4 \%$ paraformaldehyde (PFA), permeabilized with $0.3 \%$ Triton X-100 (Sigma-Aldrich, X100) in PBS and blocked with 10\% goat serum for 10 min at room temperature. Thereafter, samples were incubated with primary antibodies overnight at $4{ }^{\circ} \mathrm{C}$ and with secondary antibodies for $2 \mathrm{hrs}$ at $37^{\circ} \mathrm{C}$. For immunohistochemistry analyses, the brains were gained after perfusing with $4 \%$ PFA, post-fixed in $4 \%$ PFA for $12 \mathrm{~h}$ and dehydrated gradiently in sucrose $(15 \%$ and $30 \%$ ) until they sank to the bottom. Then, brains were frozen in OCT embedding medium at $-20^{\circ} \mathrm{C}$ for at 
least $2 \mathrm{hrs}$, and serial coronal sections with a thickness of $16 \mu \mathrm{m}$ in SVZ (bregma $1.09 \mathrm{~mm}$ to $0.13 \mathrm{~mm}$ ), $30 \mu \mathrm{m}$ in SGZ (bregma -1.07 to $-2.45 \mathrm{~mm}$ ) and $16 \mu \mathrm{m}$ in olfactory (bregma $4.57 \mathrm{~mm}$ to $4.07 \mathrm{~mm}$ ) were sliced. Samples were permeabilized with $0.3 \%$ Triton X-100 in PBS, fixated and permeabilized further with acetone at $-20^{\circ} \mathrm{C}$ for $20 \mathrm{~min}$, and blocked with $10 \%$ goat serum for $1 \mathrm{hr}$ at room temperature. Thereafter, samples were incubated with primary antibodies overnight at $4^{\circ} \mathrm{C}$ and with secondary antibodies for 2 hrs at $37^{\circ} \mathrm{C}$.

For BrdU staining, samples were denatured to expose antigen with $2 \mathrm{M} \mathrm{HCL}$ at $37^{\circ} \mathrm{C}$ for $10 \mathrm{~min}$ in cells and 16 min in tissues before the permeabilization with Triton X-100.

Antibodies utilized in above staining assays were listed in Table 2. Nuclei were counter-stained with DAPI (1 $\mathrm{\mu g} / \mathrm{ml}$, Cat\# D9542, Sigma-Aldrich).

\section{Imaging and quantitative analysis}

Images were captured by a fluorescent microscope (Model BX51, Olympus, Tokyo, Japan) or a SP8 confocal microscope (Leica Microsystems, Wetzlar, Germany) depending on the requirements. Staining was quantitatively analyzed by a double-blinded manner using MetaMorph software (Molecular Devices, Sunnyvale, CA) from at least three independent experiments. In vitro, for each coverslip, 6 different nonoverlapping fields under 20 -fold magnification were randomly selected, and the percentages of $\mathrm{BrdU}^{+}$and Tuj $1^{+}$cells were calculated. In vivo, imaging and quantitative analysis for each mouse were performed as reported previously [24]. $\mathrm{Ki}^{+}, \mathrm{DCX}^{+}$and $\mathrm{BrdU}^{+} / \mathrm{NeuN}^{+}$cells were counted, and the average numbers of these immunopositive cells per section were calculated, which then multiplied the number of sections per SVZ, SGZ and olfactory bulb to get the total quantity for each mouse.

\section{Odor detection test}

To assess the influence of rapamycin on the odor detection threshold of mice, we adopted an odor detection test $[25,26]$ with some minor modifications. This test includes two phases: habituation and testing. For habituation, mice were transferred to the test chamber $(31 \times 25 \times 12.5 \mathrm{~cm})$ to acclimatize to the environment for $30 \mathrm{~min}$ per time during the four consecutive times. During a 3-min testing period, each mouse in the test chamber was exposed to two odors on two separate filter papers, one of which was water and the other was different concentrations of novel odor (coconut powder, concentration: 1 $\mathrm{mg} / \mathrm{ml}, 10 \mathrm{mg} / \mathrm{ml}$ and $100 \mathrm{mg} / \mathrm{ml}$ ). Sniffing time was measured for three concentrations tested in separate 3 days, in an ascending order. If animals can detect the novel odor was determined by the ratio of the time spent sniffing novel odor to the total sniffing time. $50 \%$ of sniffing duration was considered that mice were able to detect olfactory stimulus.

\section{Olfactory avoidance test}

The olfactory avoidance test was performed using a component of fox feces, nTMT ( nondehydrogenated 2,4,5-trimethylthiazole ), to investigate the influence of rapamycin on the odor 
detection ability of mice $[27,28]$. Prior to the test, mice were habituated to the experimental environment, in which mice were placed in a test cage $(31 \times 25 \times 12.5 \mathrm{~cm})$ for 30 min per time during the four consecutive times, under the weak-light condition ( $<5$ lux). In the testing phase, the test cage was divided into two equal areas and three different volumes $(0,0.1$, and $1 \mu \mathrm{l})$ of undiluted nTMT was dropped on the filter paper $(2 \mathrm{~cm} \times 2 \mathrm{~cm})$ in every side of test cage. Then, mouse was placed on the opposite side of the test cage and the behavior was recorded with a video camera during the 10-min test duration. There was an interval of $24 \mathrm{~h}$ among different volumes of nTMT. Avoidance time was defined as the time spent in an area without a filter paper scented with nTMT. Avoidance behavior was determined by an avoidance index as follows: avoidance index $=(P-50) / 50$, where $P$ is the percentage of avoidance time during the 10 min test period.

\section{Y-Maze test}

To assess the hippocampus-dependent spatial recognition and memory performance of the mice with rapamycin intraperitoneally injection, Y-Maze was performed as previously described [24]. Briefly, mice were transferred to the behavior room for at least 30 minutes for environment habituation. In testing stage, each mouse was placed at the end of one arm and allowed to explore freely through the three arms for $5 \mathrm{~min}$. The number of arm entries and alternations were recorded using the Smart 3.0 Video Tracking System (Harvard Apparatus, Cambridge, Massachusetts). An arm entry is defined as when all four paws are completely within the arm. Spontaneous alternation is referred to that mouse entries all three arms in the overlapping triplet sets. The alternation percentage was calculated as follows: [ spontaneous alternation / ( total number of arm entries-2)]×100.

\section{Novel object recognition test}

The novel object recognition (NOR) test was performed, as previously reported [24], to evaluate the hippocampus-dependent non-spatial memory retention for the mice with rapamycin injection. It was performed in an open field area ( $40 \times 40 \times 35 \mathrm{~cm}$ test box) with two different kinds of objects. Both objects are generally consistent in height and volume, but are different in shape and appearance. This test includes three phases: habituation, training, and testing. First, mouse was habituated to the test box for 30 minutes daily for 3 consecutive days. In the training phase, mouse was allowed to explore two identical objects placed at a symmetric position of the box for 10 minutes. $1.5 \mathrm{hr}$ later, one object was replaced by a novel object, and the animal was allowed to explore freely for 5 minutes. The time spent exploring each object in the training and testing phases was recorded using the Smart 3.0 Video Tracking System (Harvard Apparatus, Cambridge, Massachusetts). An exploration was considered as when mouse nasal tip was less than $2 \mathrm{~cm}$ from the object. The object preference index was defined as the percentage of time spent exploring any one of the two objects (training session) or the novel one (retention session) over the total time exploring both objects.

\section{Statistical analysis}


All data in this study were analyzed using SPSS Software and presented as mean \pm standard deviations (SD). The unpaired Student's t-test was used for comparisons between two groups, and One-Way ANOVA with Tukey's post-hoc test was used for multi-group comparisons. The statistically significant level was determined by $p<0.05$.

\section{Results}

\section{Compromised autophagy activity of NPCs with aging.}

To clarify the activity of autophagy in NPCs in different aging stage, LC3B-II in homogenates from the SVZ and the SGZ of hippocampal dentate gyrus was detected with Western Bloting. As shown in Fig. 1A, the level of LC3B-II was decreased in the SVZ and SGZ homogenates from middle-aged mice compared to that from neonatal ones. Similarly, a 40\% reduction of LC3B-II was observed in cultured NPCs from middle-aged mice (Fig. 1B). To further monitor autophagy activity, NPCs from GFP-LC3 mice were treated with $10 \mathrm{nM}$ lysosomal degradation blocker, bafilomycin $A_{1}$, for 4 h to capture GFP-LC3 puncta $[29,30]$. As shown in Fig. 1B, bright puncta were formed in the cytoplasm of NPCs from neonatal mice, but rare in that from middle-aged mice. It is well-established that the autophagy-related genes (Atgs) are required for the formation of the isolation membrane and the autophagosome, which involves two major steps: nucleation and elongation of the isolation membrane. Among these genes, Beclin 1 is essential for the nucleation of isolation membrane, and $\operatorname{Atg} 7, \operatorname{Atg} 3$ and $\operatorname{Atg} 5$ are required for the elongation of isolation membrane [31]. To further explore the relationship between autophagy and self-renewal of NPCs with aging, we assessed the expression of these Atgs in SVZ and SGZ homogenates, as well as in cultured NPCs from neonatal, young and middle-aged mice by qRT-PCR. Both in vivo and in vitro data indicated the mRNA levels of Beclin 1, Atg7, Atg3 and Atg5 were dramatically decreased in homogenates and cultured NPCs from young and middle-aged mice (Fig. 1C). It is reasonable to postulate that the impaired

autophagy may be involved in the decline of the viability of adult NPCs, which was previously reported by our group and others $[8,32,33]$.

\section{Regulation of self-renewal and differentiation of NPCs by autophagy.}

To validate the role of autophagy in maintaining NPCs proliferation and differentiation, neonatal NPCs were transfected with siRNA that targeted the Atg7gene, a key component in the formation of autophagosome and serving as an E1-like activating enzyme required for the conversion of LC3B-I to LC3B-II [34-36]. The ATG7 protein and Atg7 mRNA level were efficiently down-regulated after transfection with the Atg7 specific siRNA ( si-Atg7) (Fig. 2A). Concomitantly, LC3B-囚 level also reduced and p62 level was elevated, indicating impaired autophagy (Fig. 2B). Meanwhile, knocking down Atg 7 suppressed neonatal NPCs proliferation, characterized by forming fewer and smaller neurospheres (Fig. 2C) and significantly down-regulated BrdU incorporation (Fig. 2D). As shown in Fig. 2E, neonatal NPCs with Atg7 knockdown also displayed the senescence-like phenotype, with increased $\beta$-galactosidase signal. To further investigate whether si-Atg7-induced autophagic dysfunction affected the neuronal differentiation of neonatal NPCs, cells transfected with either scramble siRNA or si-Atg7 were cultured in the 
differentiation medium for 5 days and quantified the percentage of cells expressing immature neuronal marker Tuj1. As illustrated in Fig. 2F, compromised neuronal differentiation was found in neonatal NPCs with Atg7 knockdown. Taken together, these data demonstrate the correlation between the autophagy dysfunction and impaired self-renewal and differentiation of NPCs, suggesting that activation of autophagy may be a feasible strategy to counteract the down-regulation of self-renewal in adult NPCs.

Given that compromised autophagy recapitulates the phenotypes of aging NPCs, we wished to know if enhanced autophagy could stimulate the potency of NPCs. We applied rapamycin, a well-known autophagy inducer [37] to the middle-aged NPCs and observed the autophagic level as well as NPCs potency. As shown in Fig. 3A, rapamycin (50nM) application increased GFP-LC3 puncta. The western blotting results showed that rapamycin-stimulated NPCs had an increased level of LC3B-II, along with attenuated p62, which was selectively degraded by autolysosome in downstream steps of autophagosome formation [34,38,39] (Fig. 3B). It was reported that rapamycin triggered the autophgy via mTOR-ULK pathway [40]. To confirm this, we detected the levels of phosphorylated mTOR and ULK1. As displayed in Fig. 3B, the phosphorylation levels of mTOR at Ser 2448 and ULK1 at Ser757 were significantly declined in the rapamycin-treated NPCs compared to control. In line with these findings, rapamycin-treated middle-aged NPCs formed more neuropheres with larger size (Fig. $3 \mathrm{C}$ ) and incorporated more BrdU than control (Fig. 3D). Moreover, rapamycin treatment increased Tuj $1^{+}$cells (Fig. 3F) and decreased SA- $\beta$-gal positive cells (Fig. 3E) in a concentration-dependent manner in middle-aged NPCs. Those results suggest that pharmacological activation of autophagy rejuvenate the viability and pluripotency of middle-aged NPCs.

\section{Rapamycin induced in vivo neurogenesis via activating autophagy.}

Encouraged by the in vitro results, we sought to determine whether in vivo administration of rapamycin could ameliorate the decline of autophagy in the brain and facilitate adult neurogenesis. To confirm this, 7-8 month-old mice were intraperitoneally injected with rapamycin $(4 \mathrm{mg} / \mathrm{kg})$ every other day for 4 weeks (Fig. 4A and 5A) according to previous reports [41]. As displayed in Fig. 4B, rapamycin elevated endogenous autophagy activity was observed within the SVZ, a main neurogenic niche, as represented by

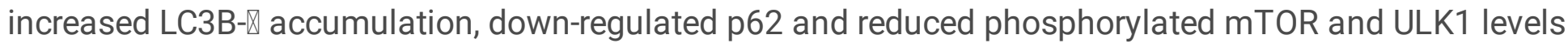
in rapamycin group. In vivo NPCs proliferation and neural differentiation were determined by endogenous proliferation marker Ki67 and immature neuron-specific marker doublecortin (DCX). Notably, the numbers of $\mathrm{Ki} 67^{+}$and $\mathrm{DCX}^{+}$cells were significantly increased in rapamycin-treated mice ( Fig. 4C-D ). Since newly formed neurons in the SVZ can migrate and integrate into the olfactory bulb circuits [2], we next examined the olfactory bulb neurogenesis with BrdU through six-day consecutive injections. The number of $\mathrm{NeuN}^{+} / \mathrm{BrdU}^{+}$colabeled cells in the granule cell layer $(\mathrm{GCL})$ of the $\mathrm{OB}$ in rapamycin group was significantly higher than that of control (Fig. 4E). The olfactory behavior was examined using odor detection test and olfactory avoidance test. Rapamycin-treated mice were able to detect lower odor concentration $(10 \mathrm{mg} / \mathrm{ml})$, whereas DMSO control mice only detected an odor at $100 \mathrm{mg} / \mathrm{ml}$, indicating improved detection sensitivity after rapamycin application (Fig. 4F). Furthermore, we performed an olfactory avoidance test using nTMT which was reported to evoke fear responses [27,28]. Interestingly, 
when a lower amount $(0.1 \mu \mathrm{l})$ of nTMT was used, rapamycin-treated mice showed significant avoidance responses compared with DMSO control mice, but no difference to a high amount of nTMT (1 $\mu$ l) (Fig. 4G), indicating that rapamycin-treated mice have increased sensitivity to detect odorants. These observations imply that activation of autophagy by rapamycin can enhance neurogenesis in the SVZ/OB and improve olfactory sensitivity in middle-aged mice.

The other major site of neurogenic niche in the brain is the hippocampus where NPCs predominantly accumulated within the SGZ of dentate gyrus [5]. We wondered whether intraperitoneal injection of rapamycin also stimulates adult neurogenesis in middle-aged SGZ. As expected, rapamycin administration significantly increased the expression of LC3B-II in DG, while reduced the levels of p62, phosphorylated mTOR and ULK1. These data showed that rapamycin raised the autophagic level of middle-aged hippocamus. Concurrently, there were more proliferative NPCs and new neurons in SGZ in rapamycin group than those in control group, as evidenced by $\mathrm{BrdU}^{+}$and $\mathrm{DCX}^{+}$staining (Fig. $\mathbf{~ C ~ a n d ~ D ) . ~}$ Further, rapamycin-treated mice showed improved spatial working memory as documented by the Y-Maze test. Compared to DMSO controls, rapamycin-treated mice obtained approximately $10 \%$ higher spontaneous alternation (Fig. 5E, left panel), while there was no significant difference for exploratory activities between the two groups, as indicated by the total numbers of arm entries (Fig. 5E, right panel). To evaluate learning and memory, we next performed the NOR test, which was based on the natural tendency of rodents to investigate new objects. During the training phase, the time percentages spent exploring the two objects were the same between rapamycin-treated and DMSO mice (Fig. 5F, left), indicating the approximately similar levels of motivation and curiosity to the novel object. However, in the testing phase, rapamycin-treated mice spent more time with novel objects than familiar ones, whereas DMOS control mice showed no such difference (Fig. 5F, right). These data suggest that activation of autophagy by rapamycin promotes the new neurons generation and functionally improves cognitive ability in middle-aged mice.

\section{Discussion}

Adult neurogenesis is of great importance in maintaining normal brain function and repairing neuronal damage [42,43]. Understanding how adult neurogenesis is regulated attracts the attention of neuroscientists. In the present study, we have demonstrated that autophagy plays a pivotal role in adult neurogenesis. With aging, the expression and activity of autophagy-related proteins in middle-aged SVZ/SGZ and cultured NPCs declined significantly. Inhibiting Atg7 by siRNA impaired the pluripotency of NPCs. Conversely, activating autophagy by rapamycin revitalized NPCs' viability and pluripotency. In vivo, injection of rapamycin stimulated neurogenesis in middle-aged SVZ/SGZ as well as functional recovery such as improved olfactory sensitivity and cognitional capacities. Consequently, drugs explorations or other interventions targeting the activation of endogenous neurogenesis will be of great utility, potentially serving as effective strategies for the prevention and treatment of age-related brain dysfunction.

Many factors, including diet, exercise, hormones, neurotransmitters, have been reported to affect adult neurogenesis [44-46]. Nevertheless, the mechanism intrinsically underlying adult neurogenesis remains to 
be illustrated. Protein homeostasis is the precondition to maintain cellular normal function, such as neurogenesis. Our previous work has shown that proteasome dysfunction leads to declined NPCs proliferation and differentiation [8]. Autophagy, as the major alternative protein degradation pathway, plays a vital role in brain development and function. During the developmental stage, constitutive autophagy is essential for embryonic neurogenesis [47]; Impaired autophagy influences the neuron differentiation and proliferation of NPCs, which leads to severe neural tube defects [48-50]. At early postnatal stages, basal autophagy is required for maintaining the viability and pluripotency of NSCs/NPCs [13-15]. Compromised autophagy is also implicated in the pathogenesis of neurodegenerative diseases [51,52], among which neurogenesis is likely affected.

In present study, we found that the expression of Atg3, Atg5, Atg7, Beclin 1 and LC3II in SVZ and hippocampus of middle-aged mice decreased, compared with that in neonatal mice, which is accompanied by declined adult neurogenesis. These observations were further supported by our in vitro data from cultured NPCs. Consistently, other researchers have reported deletions of Fip200, a gene essential for autophagy induction, and Atg5 result in progressively loss of adult NSCs/NPCs and deteriorated neuronal differentiation [13,15]. Among these Atgs declined in middle-aged group, the decrease of Atg7 was particularly significant. Atg7 level in middle-aged NPCs dropped approximately $72 \%$ of that in neonatal NPCs. We further demonstrated that inhibition of Atg 7 by siRNA in neonatal NPCs suppressed autophagy activity as well as cell proliferation and differentiation, a phenomenon that is confirmed by the smaller neurospheres observed, less $\mathrm{BrdU}^{+}$and Tuj $1^{+}$cells, along with increased $\beta$ galactosidase. In line with our findings, Atg7 deficiency has been also implicated in neuronal loss, axonal degeneration and memory impairment [16,53,54]. Besides that, higher Atg7 level was essential for the neuronal differentiation of the embryonic olfactory bulb [18]. Noteworthily, in some pathological conditions, high expression of $A \operatorname{tg} 7$ is also involved in inflammation and microglia activation, which are associated with cell death and childhood neurological disorders [34,55,56]. Thus, enhanced autophagy might not always be beneficial, and further investigations utilizing different animal models will be needed to elucidate the precise role of Atgs-mediated autophagy in neurogenesis.

Autophagy could also be modulated by certain compounds. Rapamycin, a well-established autophagy activator, promotes autophagic activation through inhibiting the mTOR pathway [41]. Here, we demonstrated that in vivo administration of rapamycin could ameliorate the decline of autophagy in the middle-aged brain and facilitate adult neurogenesis through autophagy activation via the suppression of mTOR. We showed that autophagic activation by rapamycin not only facilitated the endogenous neurogenesis in the middle-aged SVZ and hippocampus, but also improved the olfactory sensitivity and cognitive capability in middle-aged mice. We further confirmed in vitro that rapamycin-triggered autophagy activation via mTOR suppression rejuvenated the viability and pluripotency of middle-aged NPCs. Reports from other groups also support our observations. Singh et al. have found that activating autophagy through oral administration of rapamycin plays a neuroprotective role by alleviating the oxidative damage, inflammatory response and cell death in the aged brain [21]. Spilman et al. have 
reported that rapamycin administration protects hippocampus neurons against injury and improves the learning and memory ability through mTOR inhibition in AD mice [40].

In summary, our results revealed that autophagy was closely correlated with adult neurogenesis. Genetic suppression of Atg7 compromised neurogenesis; conversely, pharmacological activation of autophagy enhanced middle-aged neurogenesis and restored middle-aged brain function. This shed light on the regulation of adult neurogenesis. Genetic or pharmacological activation of autophagy could serve as an attractive therapeutic strategy for age-related brain dysfunction and neurodegenerative diseases where neuogeneis is compromised.

\section{Declarations}

\section{Funding}

This work was supported by National Natural Science Foundation of China $(81200254,81571381)$, Research Project Supported by Shanxi Scholarship Council of China (2020-085) and Natural Science Foundation of Shanxi Province (2014-033).

\section{Ethics approval}

All animal studies were approved by the Committee for Animal Care and Ethical Review at Shanxi Medical University.

\section{Consent to participate}

Not applicable.

\section{Consent for publication}

Not applicable.

\section{Availability of data and material}

Not applicable.

\section{Code availability}

Not applicable.

\section{Authors' contributions}

N.Y., XQ.L., XJ.N., XQ.W. and R.J. perfomed and analyzed the experiments. N.Y.and JR.W. helped in some animal experiments. KL. L. and L.L. conceived and designed the research. The manuscript was written by XQ.L., L.L. and CW. Z. 
All authors read and approved the final manuscript.

\section{Competing Interests}

The authors declare that they have no competing interests.

\section{References}

1. Gage, F. H. (2000). Mammalian neural stem cells. Science, 287(5457), 1433-1438.

2. Zhao, C., Deng, W., \& Gage, F. H. (2008). Mechanisms and functional implications of adult neurogenesis. Cell, 132(4), 645-660.

3. Seki, T., Hori, T., Miyata, H., Maehara, M., \& Namba, T. (2019). Analysis of proliferating neuronal progenitors and immature neurons in the human hippocampus surgically removed from control and epileptic patients. Sci Rep, 9(1), 18194.

4. Ho, N. F., Hooker, J. M., Sahay, A., Holt, D. J., \& Roffman, J. L. (2013). In vivo imaging of adult human hippocampal neurogenesis: Progress, pitfalls and promise. Mol Psychiatry, 18(4), 404-416.

5. Kumar, A., Pareek, V., Faiq, M., Ghosh, S., \& Kumari, C. J. I. i. c. n. (2019). Adult neurogenesis in humans: A review of basic concepts, history, current research, and clinical implications. Innov Clin Neurosci, 16, 30-37.

6. Sorrells, S. F., Paredes, M. F., Cebrian-Silla, A., Sandoval, K., Qi, D., Kelley, K. W., James, D., Mayer, S., Chang, J., Auguste, K. I., Chang, E. F., Gutierrez, A. J., Kriegstein, A. R., Mathern, G. W., Oldham, M. C., Huang, E. J., Garcia-Verdugo, J. M., Yang, Z., \& Alvarez-Buylla, A. (2018). Human hippocampal neurogenesis drops sharply in children to undetectable levels in adults. Nature, 555(7696), 377-381.

7. Romine, J., Gao, X., Xu, X., So, K., \& Chen, J. J. N. o. a. (2015). The proliferation of amplifying neural progenitor cells is impaired in the aging brain and restored by the mtor pathway activation. Neurobiol Aging, 36(4), 1716-1726.

8. Zhao, Y., Liu, X., He, Z., Niu, X., Shi, W., Ding, J. M., Zhang, L., Yuan, T., Li, A., Yang, W., \& Lu, L. (2016). Essential role of proteasomes in maintaining self-renewal in neural progenitor cells. Sci Rep, 6 , 19752.

9. Kalamakis, G., Brune, D., Ravichandran, S., Bolz, J., Fan, W., Ziebell, F., Stiehl, T., Catala-Martinez, F., Kupke, J., Zhao, S., Llorens-Bobadilla, E., Bauer, K., Limpert, S., Berger, B., Christen, U., Schmezer, P., Mallm, J. P., Berninger, B., Anders, S., Del Sol, A., Marciniak-Czochra, A., \& Martin-Villalba, A. (2019). Quiescence modulates stem cell maintenance and regenerative capacity in the aging brain. Cell, 176(6), 1407-1419 e1414.

10. Friedman, L. G., Lachenmayer, M. L., Wang, J., He, L., Poulose, S. M., Komatsu, M., Holstein, G. R., \& Yue, Z. (2012). Disrupted autophagy leads to dopaminergic axon and dendrite degeneration and promotes presynaptic accumulation of alpha-synuclein and Irrk2 in the brain. $J$ Neurosci, 32(22), 7585-7593. 
11. Fernandes, H., Hartfield, E., Christian, H., Emmanoulidou, E., Zheng, Y., Booth, H., Bogetofte, H., Lang, C., Ryan, B., Sardi, S., Badger, J., Vowles, J., Evetts, S., Tofaris, G., Vekrellis, K., Talbot, K., Hu, M., James, W., Cowley, S., \& Wade-Martins, R. J. S. c. r. (2016). Er stress and autophagic perturbations lead to elevated extracellular a-synuclein in gba-n370s parkinson's ipsc-derived dopamine neurons. Stem Cell Reports, 6(3), 342-356.

12. Hunn, B., Vingill, S., Threlfell, S., Alegre-Abarrategui, J., Magdelyns, M., Deltheil, T., Bengoa-Vergniory, N., Oliver, P., Cioroch, M., Doig, N., Bannerman, D., Cragg, S., \& Wade-Martins, R. J. C. r. (2019). Impairment of macroautophagy in dopamine neurons has opposing effects on parkinsonian pathology and behavior. Cell Rep, 29(4), 920-931.e927.

13. Wang, C., Liang, C., Bian, Z., Zhu, Y., \& Guan, J. J. N. n. (2013). Fip200 is required for maintenance and differentiation of postnatal neural stem cells. Nat Neurosci, 16(5), 532-542.

14. Yazdankhah, M., Farioli-Vecchioli, S., Tonchev, A., Stoykova, A., Cecconi, F. J. C. d., \& disease. (2014). The autophagy regulators ambra 1 and beclin 1 are required for adult neurogenesis in the brain subventricular zone. Cell Death Dis, 5, e1403.

15. Xi, Y., Dhaliwal, J., Ceizar, M., Vaculik, M., Kumar, K., Lagace, D. J. C. d., \& disease. (2016). Knockout of atg 5 delays the maturation and reduces the survival of adult-generated neurons in the hippocampus. Cell Death Dis, 7, e2127.

16. Komatsu, M., Waguri, S., Chiba, T., Murata, S., Iwata, J., Tanida, I., Ueno, T., Koike, M., Uchiyama, Y., Kominami, E., \& Tanaka, K. J. N. (2006). Loss of autophagy in the central nervous system causes neurodegeneration in mice. Nature, 441(7095), 880-884.

17. Chen, D., Pang, S., Feng, X., Huang, W., Hawley, R., \& Yan, B. J. N. I. (2013). Genetic analysis of the atg7 gene promoter in sporadic parkinson's disease. Neurosci Lett, 534, 193-198.

18. Vázquez, P., Arroba, A., Cecconi, F., de la Rosa, E., Boya, P., \& de Pablo, F. J. A. (2012). Atg5 and ambra1 differentially modulate neurogenesis in neural stem cells. Autophagy, 8(2), 187-199.

19. Revuelta, M., \& Matheu, A. (2017). Autophagy in stem cell aging. Aging Cell, 16(5), 912-915.

20. Ravikumar, B., Vacher, C., Berger, Z., Davies, J., Luo, S., Oroz, L., Scaravilli, F., Easton, D., Duden, R., O'Kane, C., \& Rubinsztein, D. J. N. g. (2004). Inhibition of mtor induces autophagy and reduces toxicity of polyglutamine expansions in fly and mouse models of huntington disease. Nat Genet, 36(6), 585-595.

21. Singh, A., Singh, S., Tripathi, V., Bissoyi, A., Garg, G., \& Rizvi, S. J. R. r. (2019). Rapamycin confers neuroprotection against aging-induced oxidative stress, mitochondrial dysfunction, and neurodegeneration in old rats through activation of autophagy. Rejuvenation Res, 22(1), 60-70.

22. Raman, L., Kong, X., \& Kernie, S. J. N. I. (2013). Pharmacological inhibition of the mtor pathway impairs hippocampal development in mice. Neurosci Lett, 541, 9-14.

23. Wang, X., Seekaew, P., Gao, X., \& Chen, J. J. e. (2016). Traumatic brain injury stimulates neural stem cell proliferation via mammalian target of rapamycin signaling pathway activation. eNeuro, 3(5).

24. Niu, X., Zhao, Y., Yang, N., Zhao, X., Zhang, W., Bai, X., Li, A., Yang, W., \& Lu, L. J. S. c. (2020). Proteasome activation by insulin-like growth factor-1/nuclear factor erythroid 2-related factor 2 
signaling promotes exercise-induced neurogenesis. Stem Cells, 38(2), 246-260.

25. Breton-Provencher, V., Lemasson, M., Peralta, M., \& Saghatelyan, A. (2009). Interneurons produced in adulthood are required for the normal functioning of the olfactory bulb network and for the execution of selected olfactory behaviors. J Neurosci, 29(48), 15245-15257.

26. Takahashi, H., Ogawa, Y., Yoshihara, S., Asahina, R., Kinoshita, M., Kitano, T., Kitsuki, M., Tatsumi, K., Okuda, M., Tatsumi, K., Wanaka, A., Hirai, H., Stern, P., \& Tsuboi, A. (2016). A subtype of olfactory bulb interneurons is required for odor detection and discrimination behaviors. $J$ Neurosci, 36(31), 82108227.

27. Fendt, M., Endres, T. J. N., \& reviews, b. (2008). 2,3,5-trimethyl-3-thiazoline (tmt), a component of fox odor - just repugnant or really fear-inducing? Neurosci Biobehav Rev, 32(7), 1259-1266.

28. Takahashi, L., Nakashima, B., Hong, H., Watanabe, K. J. N., \& reviews, b. (2005). The smell of danger: A behavioral and neural analysis of predator odor-induced fear. Neurosci Biobehav Rev, 29(8), 11571167.

29. Pugsley, H. J. M. (2017). Quantifying autophagy: Measuring Ic3 puncta and autolysosome formation in cells using multispectral imaging flow cytometry. Methods, 112, 147-156.

30. Mauthe, M., Orhon, I., Rocchi, C., Zhou, X., Luhr, M., Hijlkema, K., Coppes, R., Engedal, N., Mari, M., \& Reggiori, F. J. A. (2018). Chloroquine inhibits autophagic flux by decreasing autophagosomelysosome fusion. Autophagy, 14(8), 1435-1455.

31. Mizushima, N., Yoshimori, T., \& Levine, B. (2010). Methods in mammalian autophagy research. Cell, 140(3), 313-326.

32. Smith, L., White, C., Villeda, S. J. C., \& research, t. (2018). The systemic environment: At the interface of aging and adult neurogenesis. Cell Tissue Res, 371(1), 105-113.

33. Cheng, Z., Li, Y. Q., \& Wong, C. S. (2016). Effects of aging on hippocampal neurogenesis after irradiation. Int J Radiat Oncol Biol Phys, 94(5), 1181-1189.

34. Xie, C., Ginet, V., Sun, Y., Koike, M., Zhou, K., Li, T., Li, H., Li, Q., Wang, X., Uchiyama, Y., Truttmann, A., Kroemer, G., Puyal, J., Blomgren, K., \& Zhu, C. J. A. (2016). Neuroprotection by selective neuronal deletion of atg7 in neonatal brain injury. Autophagy, 12(2), 410-423.

35. Su, L., Luo, R., Liu, Q., Su, J., Yang, L., Ding, Y., Xu, L., \& Yao, Y. J. A. (2017). Atg5- and atg7-dependent autophagy in dopaminergic neurons regulates cellular and behavioral responses to morphine. Autophagy, 13(9), 1496-1511.

36. Donde, A., Sun, M., Jeong, Y., Wen, X., Ling, J., Lin, S., Braunstein, K., Nie, S., Wang, S., Chen, L., \& Wong, P. J. A. (2020). Upregulation of atg7 attenuates motor neuron dysfunction associated with depletion of tardbp/tdp-43. Autophagy, 16(4), 672-682.

37. Liu, Q., Zhou, X., Li, C., Zhang, X., \& Li, C. J. O. I. (2018). Rapamycin promotes the anticancer action of dihydroartemisinin in breast cancer mda-mb-231 cells by regulating expression of atg7 and dapk. Oncol Lett, 15(4), 5781-5786.

38. Bjørkøy, G., Lamark, T., Brech, A., Outzen, H., Perander, M., Overvatn, A., Stenmark, H., \& Johansen, T. J. T. J. o. c. b. (2005). P62/sqstm1 forms protein aggregates degraded by autophagy and has a 
protective effect on huntingtin-induced cell death. J Cell Biol, 171(4), 603-614.

39. Gao, W., Chen, Z., Wang, W., \& Stang, M. J. P. o. (2013). E1-like activating enzyme atg7 is preferentially sequestered into p62 aggregates via its interaction with Ic3-i. PLoS One, 8(9), e73229.

40. Spilman, P., Podlutskaya, N., Hart, M., Debnath, J., Gorostiza, O., Bredesen, D., Richardson, A., Strong, R., \& Galvan, V. J. P. o. (2010). Inhibition of mtor by rapamycin abolishes cognitive deficits and reduces amyloid-beta levels in a mouse model of alzheimer's disease. PLoS One, 5(4), e9979.

41. Li, G., Miskimen, K., Wang, Z., Xie, X., Tse, W., Gouilleux, F., Moriggl, R., \& Bunting, K. J. L. (2010). Effective targeting of stat5-mediated survival in myeloproliferative neoplasms using abt-737 combined with rapamycin. Leukemia, 24(8), 1397-1405.

42. Ming, G., \& Song, H. J. N. (2011). Adult neurogenesis in the mammalian brain: Significant answers and significant questions. Neuron, 70(4), 687-702.

43. Deng, W., Aimone, J., \& Gage, F. J. N. r. N. (2010). New neurons and new memories: How does adult hippocampal neurogenesis affect learning and memory? Nat Rev Neurosci, 11(5), 339-350.

44. Cutler, R., \& Kokovay, E. J. C. o. i. p. (2020). Rejuvenating subventricular zone neurogenesis in the aging brain. Curr Opin Pharmacol, 50, 1-8.

45. Katsimpardi, L., \& Lledo, P. J. C. o. i. n. (2018). Regulation of neurogenesis in the adult and aging brain. Curr Opin Pharmacol, 53, 131-138.

46. Livingston-Thomas, J., Nelson, P., Karthikeyan, S., Antonescu, S., Jeffers, M., Marzolini, S., \& Corbett, D. J. N. t. j. o. t. A. S. f. E. N. (2016). Exercise and environmental enrichment as enablers of taskspecific neuroplasticity and stroke recovery. Neurotherapeutics, 13(2), 395-402.

47. Fimia, G., Stoykova, A., Romagnoli, A., Giunta, L., Di Bartolomeo, S., Nardacci, R., Corazzari, M., Fuoco, C., Ucar, A., Schwartz, P., Gruss, P., Piacentini, M., Chowdhury, K., \& Cecconi, F. J. N. (2007). Ambra1 regulates autophagy and development of the nervous system. Nature, 447(7148), 1121-1125.

48. Mizushima, N., \& Levine, B. J. N. c. b. (2010). Autophagy in mammalian development and differentiation. Nat Cell Biol, 12(9), 823-830.

49. Casares-Crespo, L., Calatayud-Baselga, I., García-Corzo, L., \& Mira, H. J. F. i. c. n. (2018). On the role of basal autophagy in adult neural stem cells and neurogenesis. Front Cell Neurosci, 12, 339.

50. Wu, X., Fleming, A., Ricketts, T., Pavel, M., Virgin, H., Menzies, F., \& Rubinsztein, D. J. N. c. (2016). Autophagy regulates notch degradation and modulates stem cell development and neurogenesis. Nat Commun, 7, 10533.

51. Nikoletopoulou, V., Papandreou, M., Tavernarakis, N. J. C. d., \& differentiation. (2015). Autophagy in the physiology and pathology of the central nervous system. Cell Death Differ, 22(3), 398-407.

52. Menzies, F., Fleming, A., Caricasole, A., Bento, C., Andrews, S., Ashkenazi, A., Füllgrabe, J., Jackson, A., Jimenez Sanchez, M., Karabiyik, C., Licitra, F., Lopez Ramirez, A., Pavel, M., Puri, C., Renna, M., Ricketts, T., Schlotawa, L., Vicinanza, M., Won, H., Zhu, Y., Skidmore, J., \& Rubinsztein, D. J. N. (2017). Autophagy and neurodegeneration: Pathogenic mechanisms and therapeutic opportunities. Neuron, 93(5), 1015-1034. 
53. Komatsu, M., Wang, Q., Holstein, G., Friedrich, V., Iwata, J., Kominami, E., Chait, B., Tanaka, K., \& Yue, Z. J. P. o. t. N. A. o. S. o. t. U. S. o. A. (2007). Essential role for autophagy protein atg7 in the maintenance of axonal homeostasis and the prevention of axonal degeneration. Proc Natl Acad Sci US A, 104(36), 14489-14494.

54. Gupta, V. K., Scheunemann, L., Eisenberg, T., Mertel, S., Bhukel, A., Koemans, T. S., Kramer, J. M., Liu, K. S., Schroeder, S., Stunnenberg, H. G., Sinner, F., Magnes, C., Pieber, T. R., Dipt, S., Fiala, A., Schenck, A., Schwaerzel, M., Madeo, F., \& Sigrist, S. J. (2013). Restoring polyamines protects from age-induced memory impairment in an autophagy-dependent manner. Nat Neurosci, 16(10), 1453-1460.

55. Wang, Y., Zhou, K., Li, T., Xu, Y., Xie, C., Sun, Y., Zhang, Y., Rodriguez, J., Blomgren, K., Zhu, C. J. C. d., \& disease. (2017). Inhibition of autophagy prevents irradiation-induced neural stem and progenitor cell death in the juvenile mouse brain. Cell Death Dis, 8(3), e2694.

56. Wang, Y., Zhou, K., Li, T., Xu, Y., Xie, C., Sun, Y., Rodriguez, J., Zhang, S., Song, J., Wang, X., Blomgren, K., \& Zhu, C. J. F. i. c. n. (2019). Atg7selective neural deletion of the gene reduces irradiation-induced cerebellar white matter injury in the juvenile mouse brain by ameliorating oligodendrocyte progenitor cell loss. Front Cell Neurosci, 13, 241.

\section{Tables}

Table 1 Primers used for Real-time PCR

\begin{tabular}{lll}
\hline əne & Forward primer sequence $5^{\prime} \rightarrow 3^{\prime}$ & Reverse primer sequence $5^{\prime} \rightarrow 3^{\prime}$ \\
\hline$g 3$ & TGGTGATGGGGGATGGGTAGATAC & TTCCCCTTCATCTTCCTCGTCTTC \\
$g 5$ & GCGGTTGAGGCTCACTTTATGTCGT & TATTCCATGAGTTTCCGGTTGATG \\
$g 7$ & TCCAGTCCGTTGAAGTCCTC & CCACTGAGGTTCACCATCCT \\
’lin1 $^{\prime}$ CGCCAGGATGGTGTCTCTCGAAG & GCGGGTGATCCACATCGGT \\
zpdh & TGTGTCCGTCGTGGATCTGA & TTGCTGTTGAAGTCGCAGGAG \\
\hline
\end{tabular}

Table 2 Antibodies used in this study 


\begin{tabular}{|c|c|c|c|c|}
\hline Antibodies & Species & Application & Dilution & $\begin{array}{c}\text { Company \& Catalog } \\
\text { number }\end{array}$ \\
\hline$\overline{\text { t anti-LC3 }}$ & Rabbit & $\overline{\mathrm{WB}}$ & $1: 1000$ & Abcam, Cat\# ab51520 \\
\hline anti-p62 & Mouse & WB & 1:1000 & Abcam, Cat\# ab56416 \\
\hline t anti-ATG7 & Rabbit & WB & $1: 1000$ & $\begin{array}{l}\text { Cell } \\
\text { Technology, Cat\# } 8558\end{array}$ \\
\hline t anti-mTOR & Rabbit & WB & 1:1000 & $\begin{array}{lr}\text { Cell } & \text { Signaling } \\
\text { Technology, Cat\# } 2983\end{array}$ \\
\hline t anti-p ${ }^{S 2448}$-mTOR & Rabbit & WB & 1:1000 & $\begin{array}{l}\text { Cell } \\
\text { Technology, Cat\# } 2971\end{array}$ \\
\hline t anti-ULK1 & Rabbit & WB & 1:1000 & $\begin{array}{l}\text { Cell } \\
\text { Technology, Cat\# } 8054\end{array}$ \\
\hline t anti-p ${ }^{\text {S757-ULK1 }}$ & Rabbit & WB & 1:1000 & $\begin{array}{l}\text { Cell } \\
\text { Technology, Cat\# } 6888\end{array}$ \\
\hline anti- $\beta$-actin & Mouse & WB & 1:4000 & $\begin{array}{l}\text { Sigma-Aldrich, } \\
\text { A1978 }\end{array}$ \\
\hline t anti- $\beta$-Actin & Rabbit & WB & 1:4000 & $\begin{array}{l}\text { Sangon Biotech, Cat\# } \\
\text { A1978 }\end{array}$ \\
\hline t IgG, HRP & Rabbit & WB & $1: 5000$ & $\begin{array}{l}\text { ZSGB-BIO, Cat\# ZB- } \\
2301\end{array}$ \\
\hline IgG, HRP & Mouse & WB & 1:5000 & $\begin{array}{l}\text { ZSGB-BIO, Cat\# ZB- } \\
2305\end{array}$ \\
\hline t anti-DCX & Rabbit & IHC & $1: 200$ & $\begin{array}{l}\text { Cell Signaling } \\
\text { technology, Cat\# } 4604\end{array}$ \\
\hline t anti-Ki67 & Rabbit & IHC & $1: 400$ & Abcam, Cat\# ab15580 \\
\hline t anti-NeuN & Rabbit & IHC & $1: 400$ & $\begin{array}{l}\text { Proteintech Group, } \\
\text { Cat\# 26975-1-AP }\end{array}$ \\
\hline anti-BrdU & Mouse & IHC & $1: 200$ & Abclonal, Cat\# A1482 \\
\hline a anti-GFP & Mouse & IHC & $1: 200$ & $\begin{array}{l}\text { Cwbiotech, } \\
\text { CW0086M }\end{array}$ \\
\hline anti-Tuj1 & Mouse & $\mathrm{IHC}$ & $1: 300$ & $\begin{array}{l}\text { Sigma-Aldrich, } \\
\text { MAB1637 }\end{array}$ \\
\hline $\begin{array}{l}\text { Fluor } 555 \text { Goat anti- } \\
\text { : IgG }(\mathrm{H}+\mathrm{L})\end{array}$ & Rabbit & IHC & $1: 200$ & $\begin{array}{l}\text { Invitrogen, } \\
\text { A21429 }\end{array}$ \\
\hline $\begin{array}{l}\text { Fluor } 488 \text { Goat anti- } \\
\text { IgG }(\mathrm{H}+\mathrm{L})\end{array}$ & Mouse & IHC & $1: 200$ & $\begin{array}{l}\text { Invitrogen, } \\
\text { A28175 }\end{array}$ \\
\hline ænti-mouse Cy3 IgG & Mouse & $\mathrm{IHC}$ & $1: 400$ & $\begin{array}{lr}\text { Boster } & \text { Biological } \\
\text { Technology, } & \text { Cat\# } \\
\text { BA1031 } & \end{array}$ \\
\hline
\end{tabular}



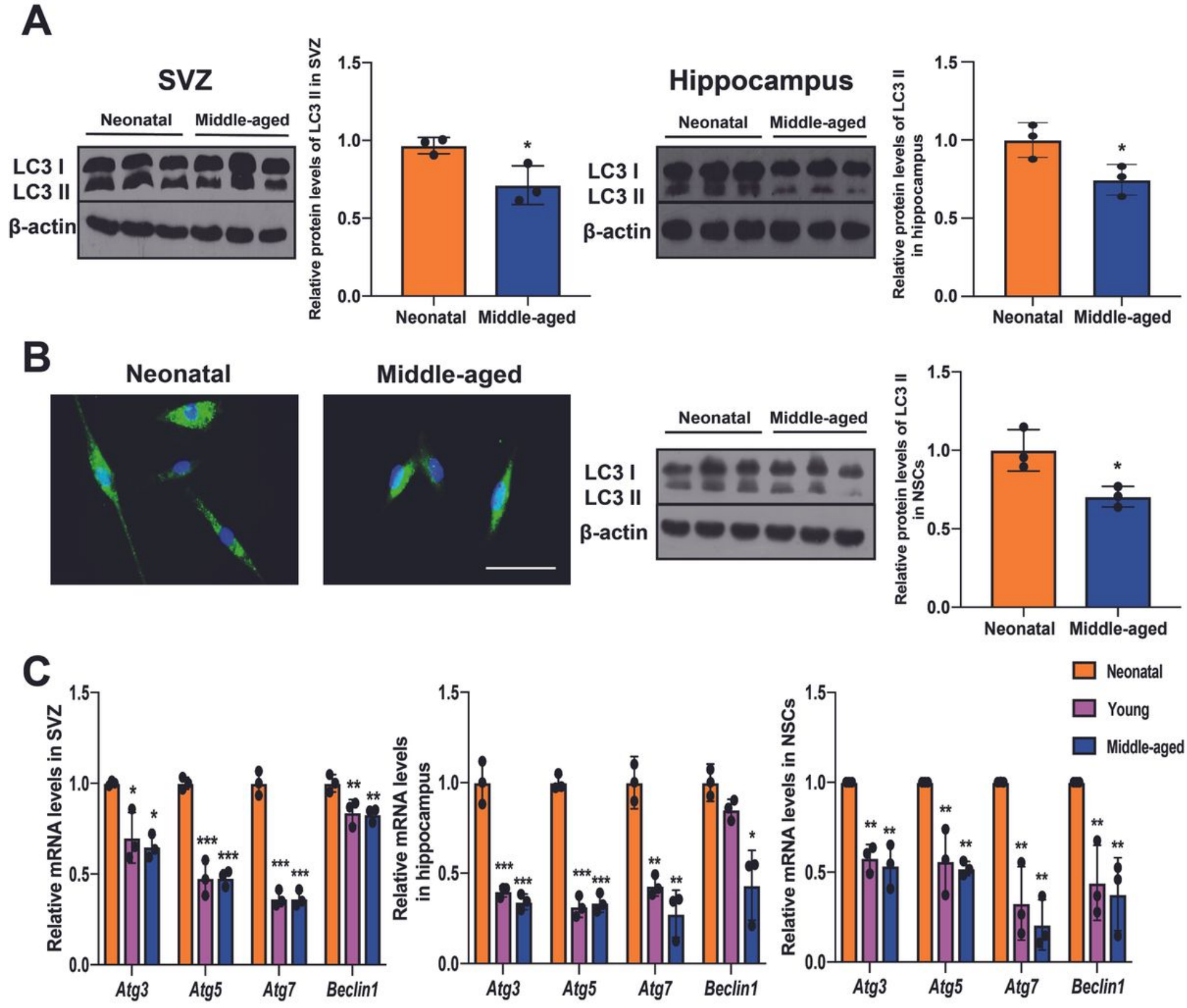

Figure 1

Compromised autophagy activity in NPCs of different aging stages. (A) Expression of LC3B- $\$ in SVZ and SGZ of neonatal and middle-aged mice $(n=3)$. (B) GFP-LC3 puncta and the expression of LC3 B- $\triangle$ in NPCs from neonatal and middle-aged mice, Scale bar=50 $\mu \mathrm{m}$. (C) The mRNA levels of autophagy-related genes (Atg7, Atg3, Atg5 and Beclin1) in SVZ, SGZ, and cultured NPCs from neonatal, young and middleaged mice $(n=3)$. Data were collected from three independent experiments, means $\pm S D .{ }^{*} p<0.05,{ }^{* \star} p<$ 0.01 and $* * * p<0.001$. 
A

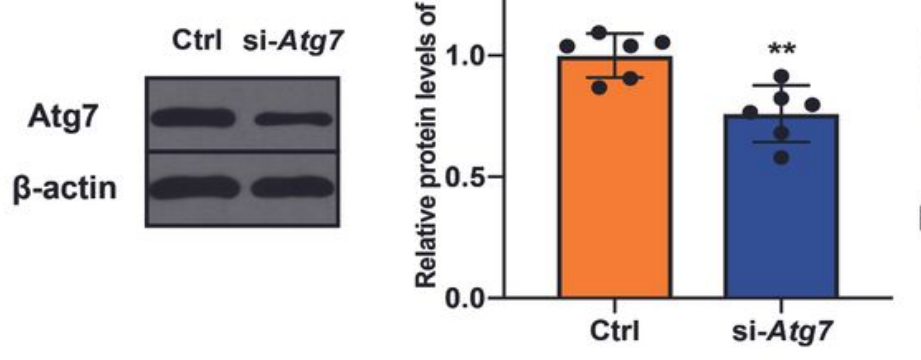

B

C
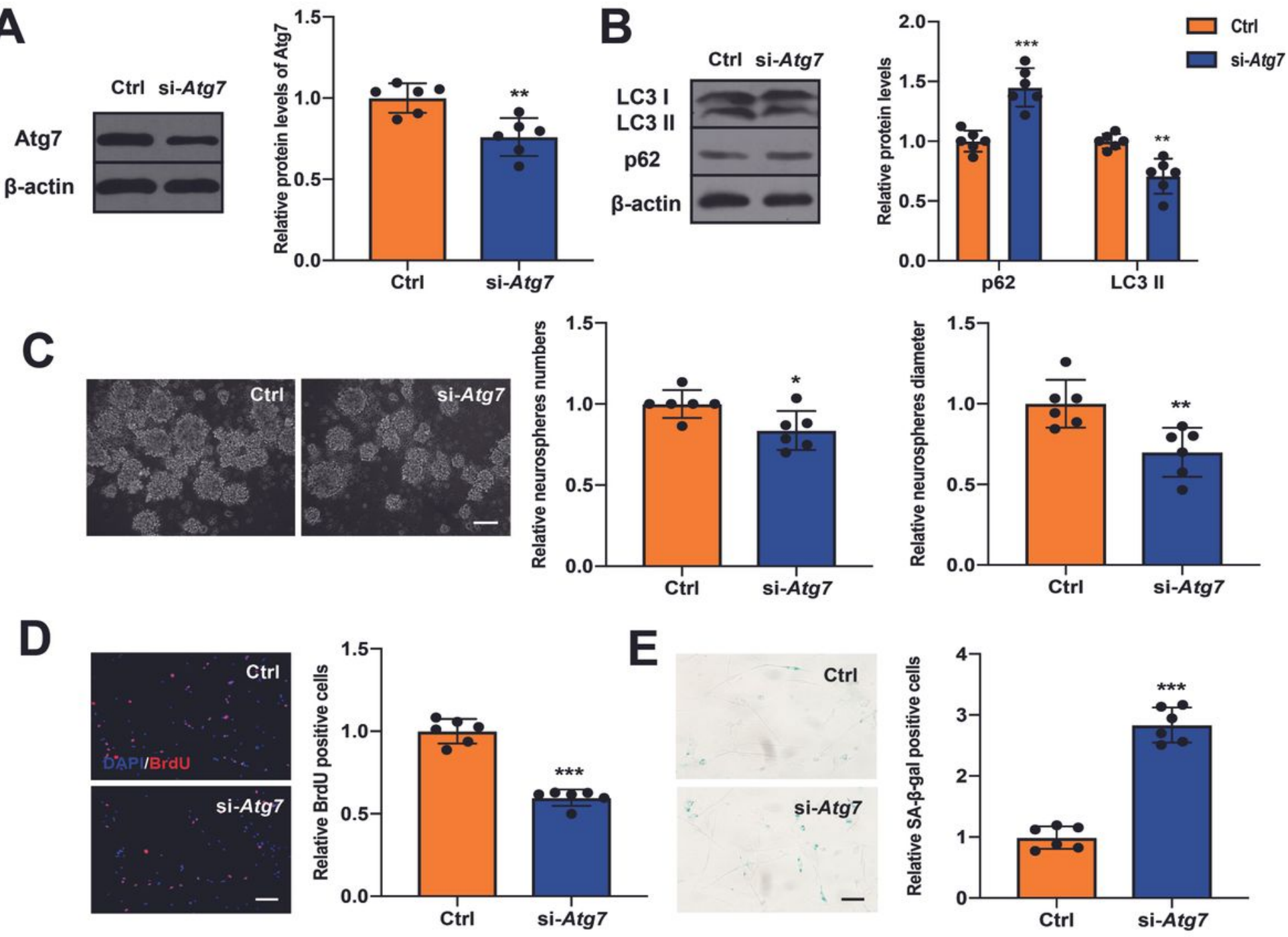

E
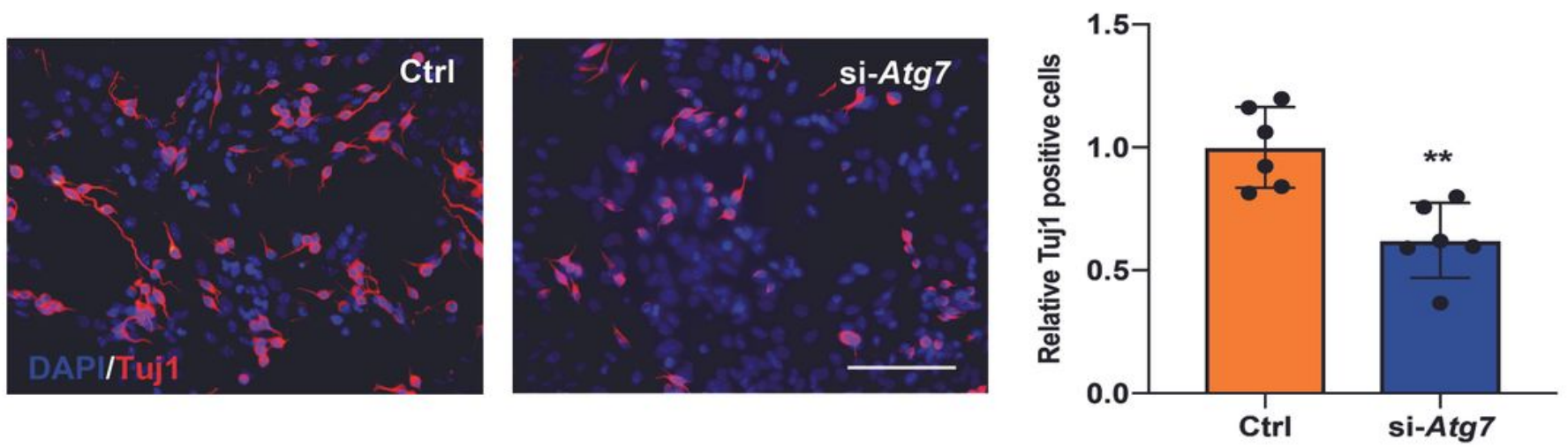

Figure 2

Atg7 gene knockdown attenuated the proliferation and differentiation capacity of neonatal NPCs. (A) siRNA inhibited the ATG7 protein expression in neonatal NPCs. (B) Atg7 knockdown induced the alteration of LC3B- $\$ and p62 in neonatal NPCs. (C) Atg7 knockdown retarded the neurospheres formation of neonatal NPCs, Scar bar $=100 \mu \mathrm{m}$. (D) Atg7 knockdown hindered the proliferation of neonatal NPCs as shown by BrdU staining, Scar bar=100 $\mu \mathrm{m}$. (E) Atg7 knockdown induced the senescence of neonatal 
NPCs, Scar bar=100 $\mu \mathrm{m}$. (F) Atg7 knockdown attenuated neuronal differentiation of neonatal NPCs as evaluated by relative Tuj1/DAPI ratio, Scar bar $=100 \mu \mathrm{m}$. Data were from six independent experiments, means \pm SD. ${ }^{*} p<0.05,{ }^{* *} p<0.01$ and ${ }^{* * *} p<0.001$.

A
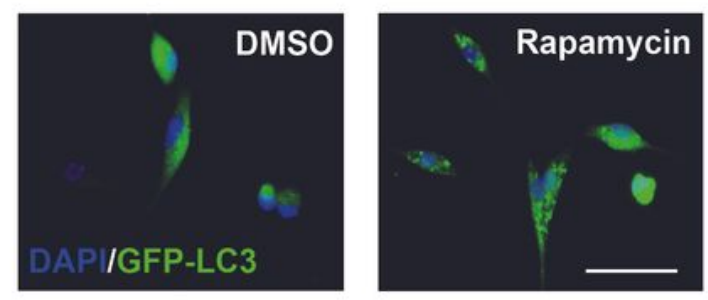

C
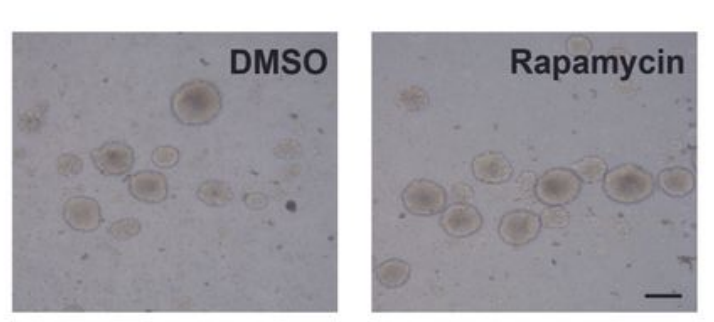

D

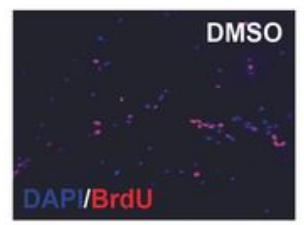

Rapamycin
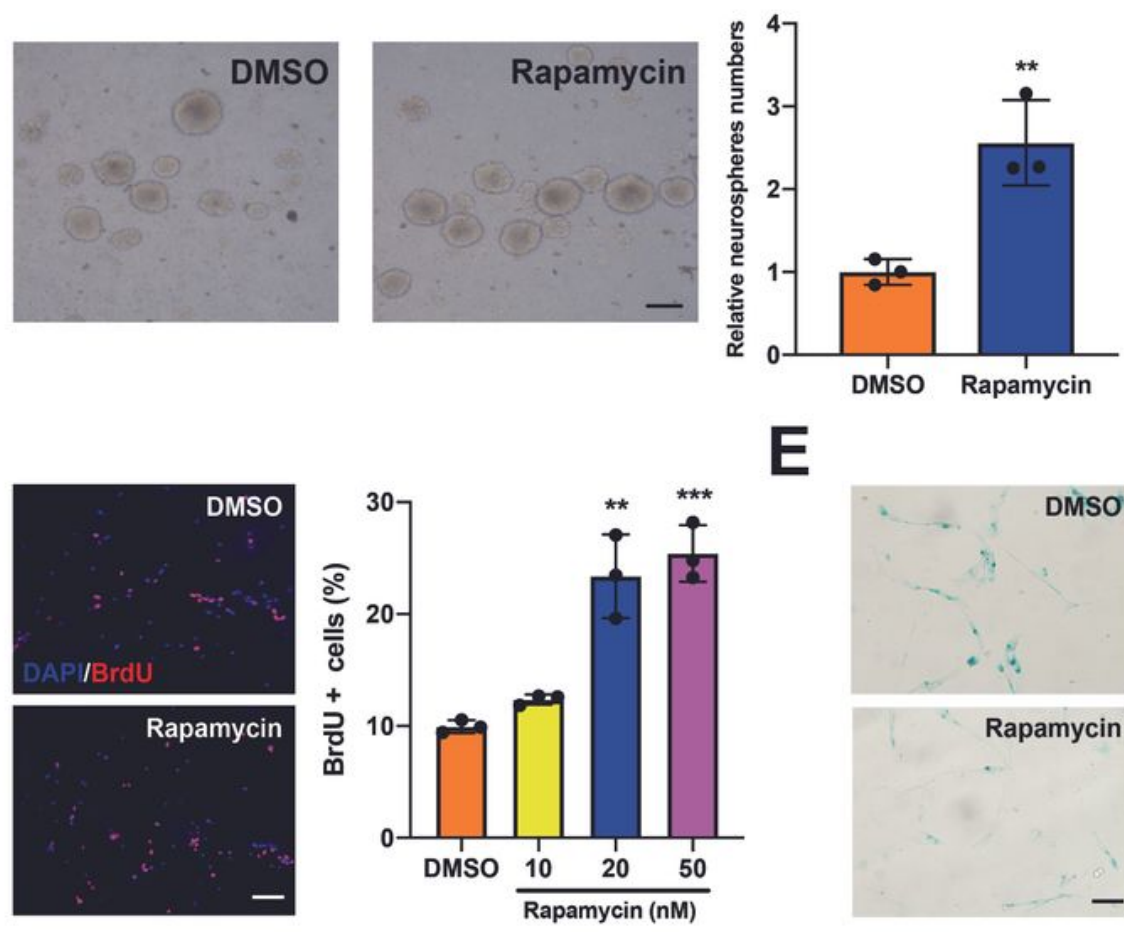

E

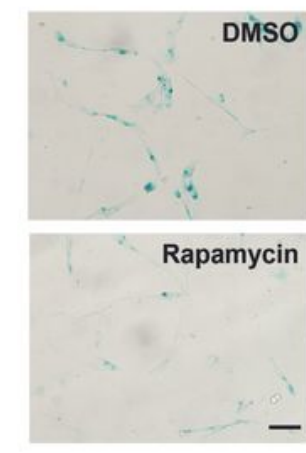

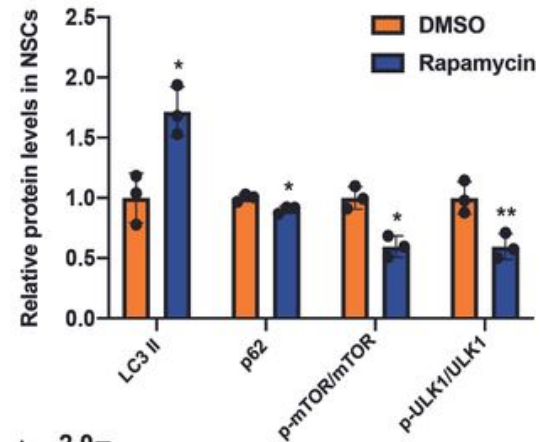
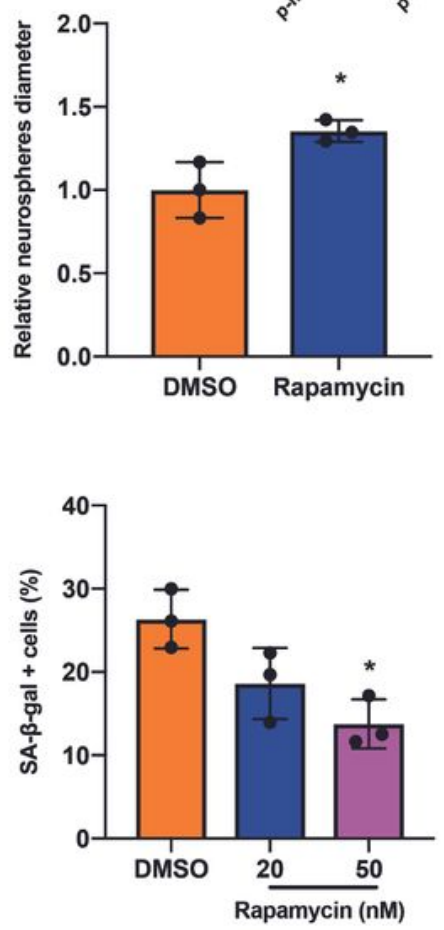

$\mathbf{F}$
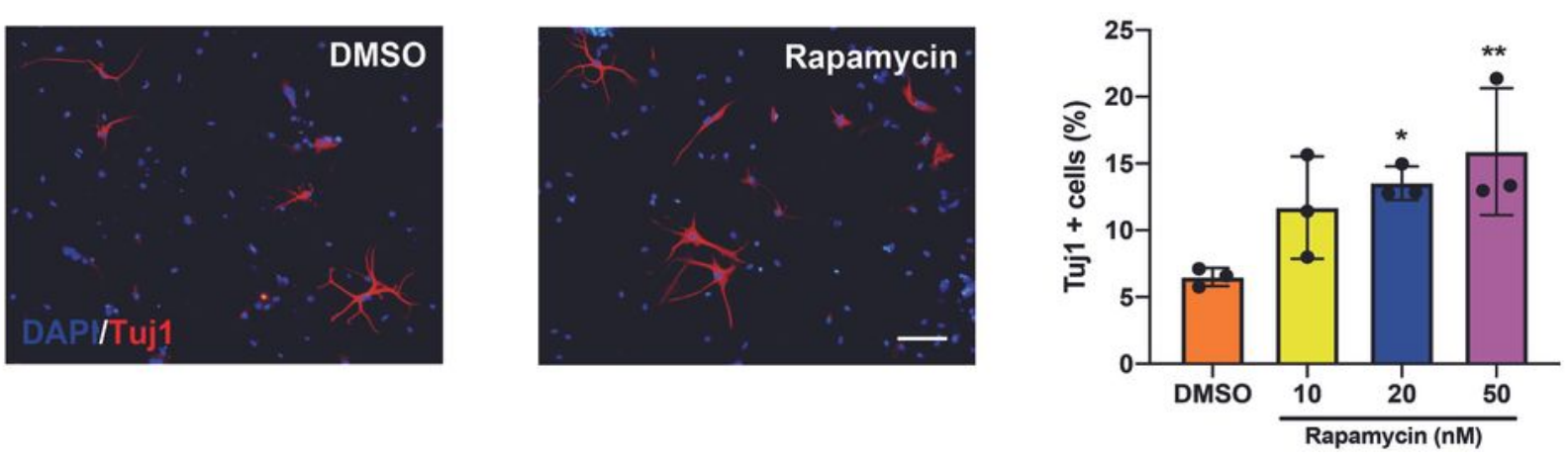

Figure 3

Rapamycin rejuvenated autophagy activity and the pluripotency of NPCs derived from middle-aged mice. (A) Rapamycin increased the number of GFP-LC3 puncta in middle-aged NPCs, Scar bar=50 $\mu \mathrm{m}$. (B) 
Rapamycin affected the expression of LC3B-II, p62, p-mTOR/mTOR and p-ULK1/ULK1 in middle-aged NPCs. (C) Rapamycin stimulated the neurospheres formation of middle-aged NPCs, Scar bar $=100 \mu \mathrm{m}$. (D) Rapamycin enhanced the proliferation of middle-aged NPCs as shown by BrdU staining. (E) Rapamycin attenuated the senescence of middle-aged NPCs, Scar bar=100 $\mu \mathrm{m}$. (F) Rapamycin increased the number of Tuj $1+$ cells in middle-aged NPCs, in a concentration-dependent manner, Scar bar $=100 \mu \mathrm{m}$. Data were from there independent experiments, means \pm SD. ${ }^{*} p<0.05,{ }^{*} p<0.01,{ }^{* \star *} p<0.001$.

A

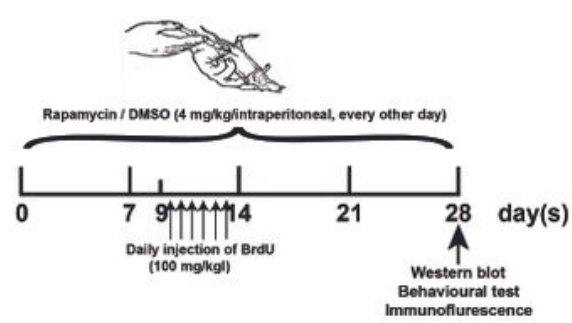

B
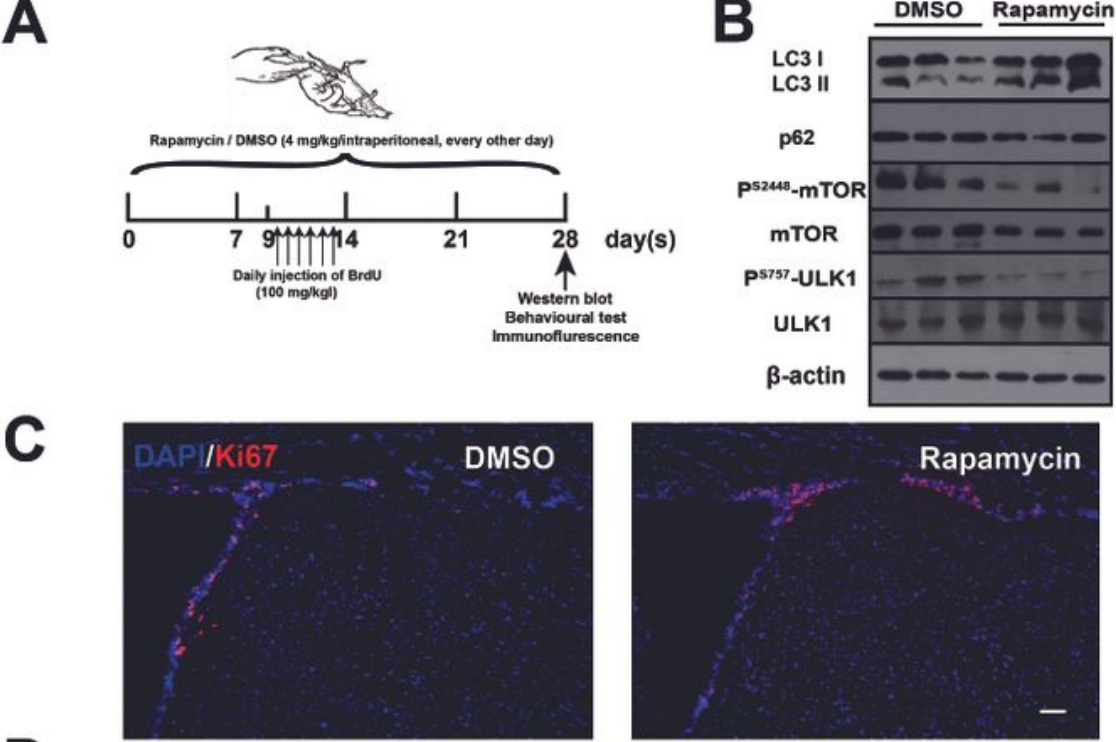

D

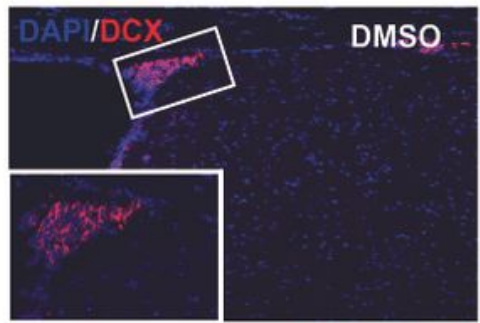

E
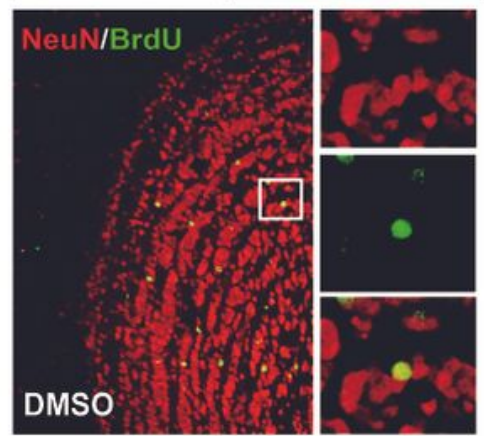
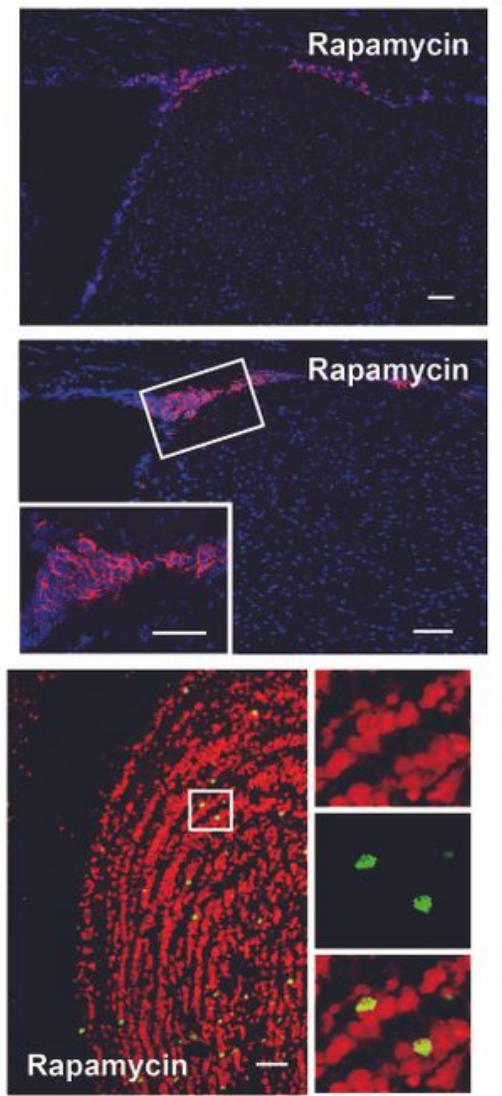
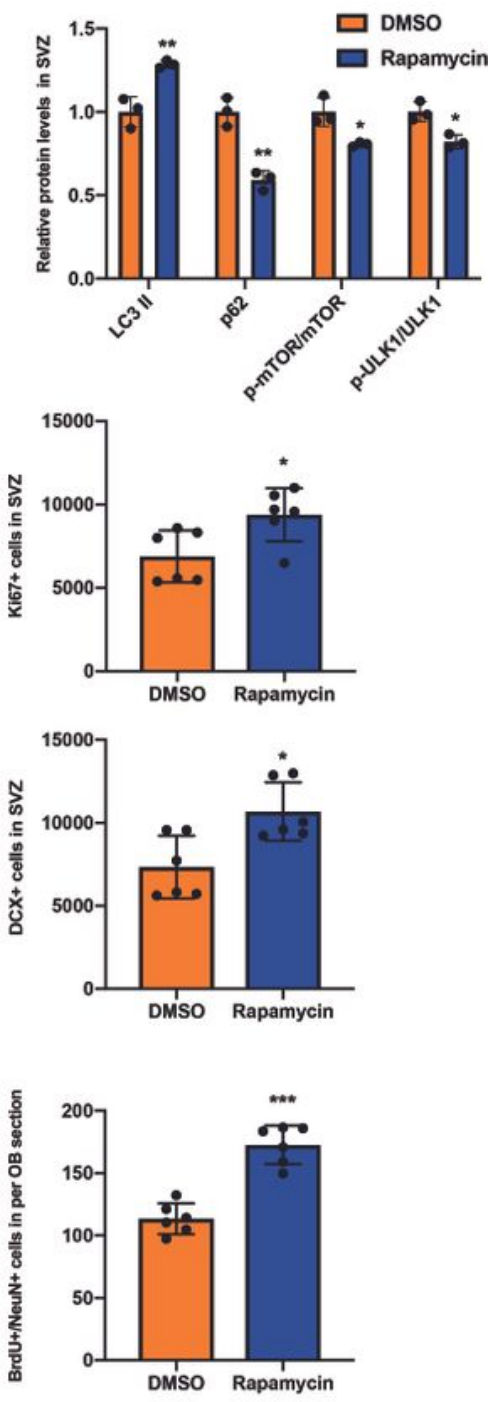

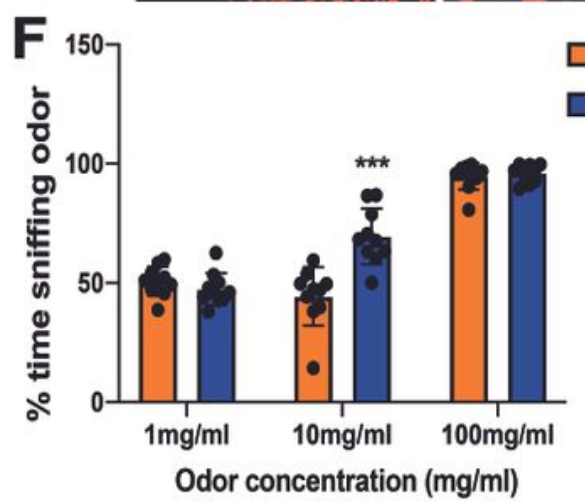

G

Rapamycin

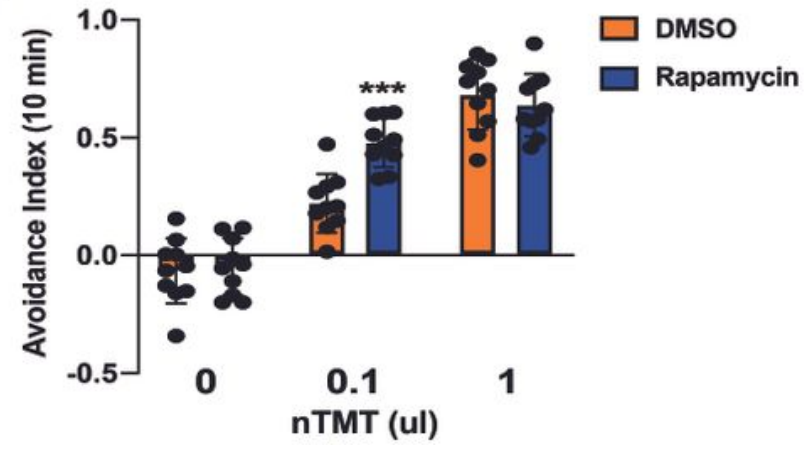

Figure 4 
Rapamycin enhanced SVZ neurogenesis and its neurofunction in middle-aged mice. (A) Schematic experimental design of rapamycin treatment in vivo and efficency evaluation. (B) Alteration of expression of LC3B-II, p62, p-mTOR/mTOR and p-ULK1/ULK1 in SVZ of middle-aged mice subjected to rapamycin injection. (C, D, E) Rapamycin stimulated neurogenesis in SVZ and olfactory bulb of middle-aged mice, as shown by Ki67, DCX, and BrdU/NeuN immunofluorescent staining respectively, Scar bar=50 $\mu \mathrm{m}$. (F, G) Rapamycin affected time for sniffing the odor and avoidance responses of middle-aged mice. $(n=10)$. Data shown are means \pm SD. ${ }^{*} p<0.05,{ }^{* *} p<0.01,{ }^{* *} \mathrm{p}<0.001$.

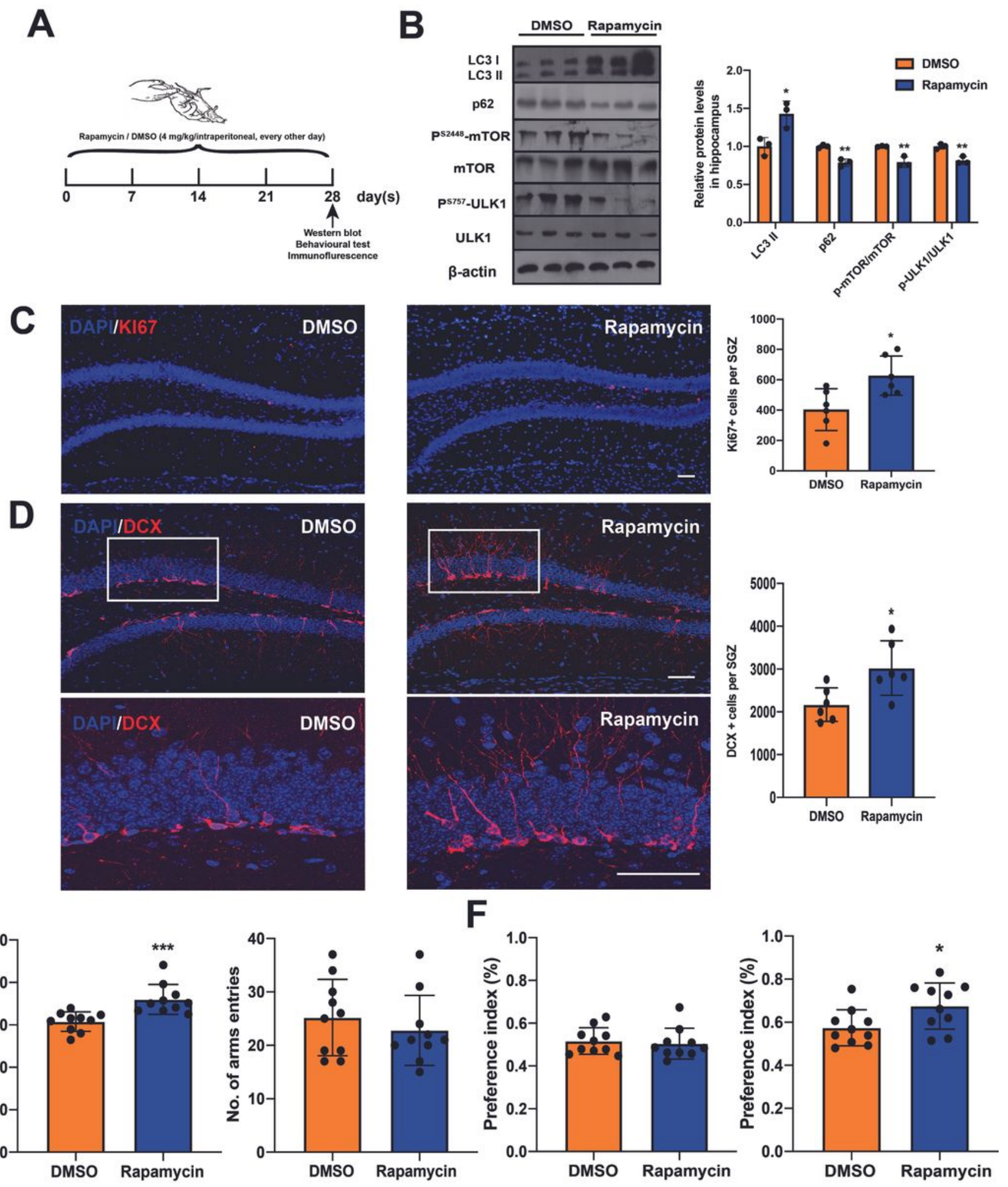




\section{Figure 5}

Rapamycin improved hippocampus neurogenesis and congnitive capability of middle-aged mice. (A) Schematic experimental design of rapamycin treatment in vivo and efficency evaluation. (B) Rapamycin affected the expression of LC3B-II, p62, p-mTOR/mTOR and p-ULK1/ULK1 in hippocampus of middleaged mice. (C, D) Rapamycin increased the Ki67, DCX positive cell number in the SGZ of the middle-aged mice, Scar bar=50 $\mu \mathrm{m}$. (E) Rapamycin improved the alternation rate in Y-Maze test of middle-aged mice. (F) Rapamycin enhanced the tendency of exploring novel objects by middle-aged mice in NOR test. Data shown are means \pm SD. ${ }^{*} p<0.05,{ }^{* *} p<0.01,{ }^{* \star *} p<0.001$. 


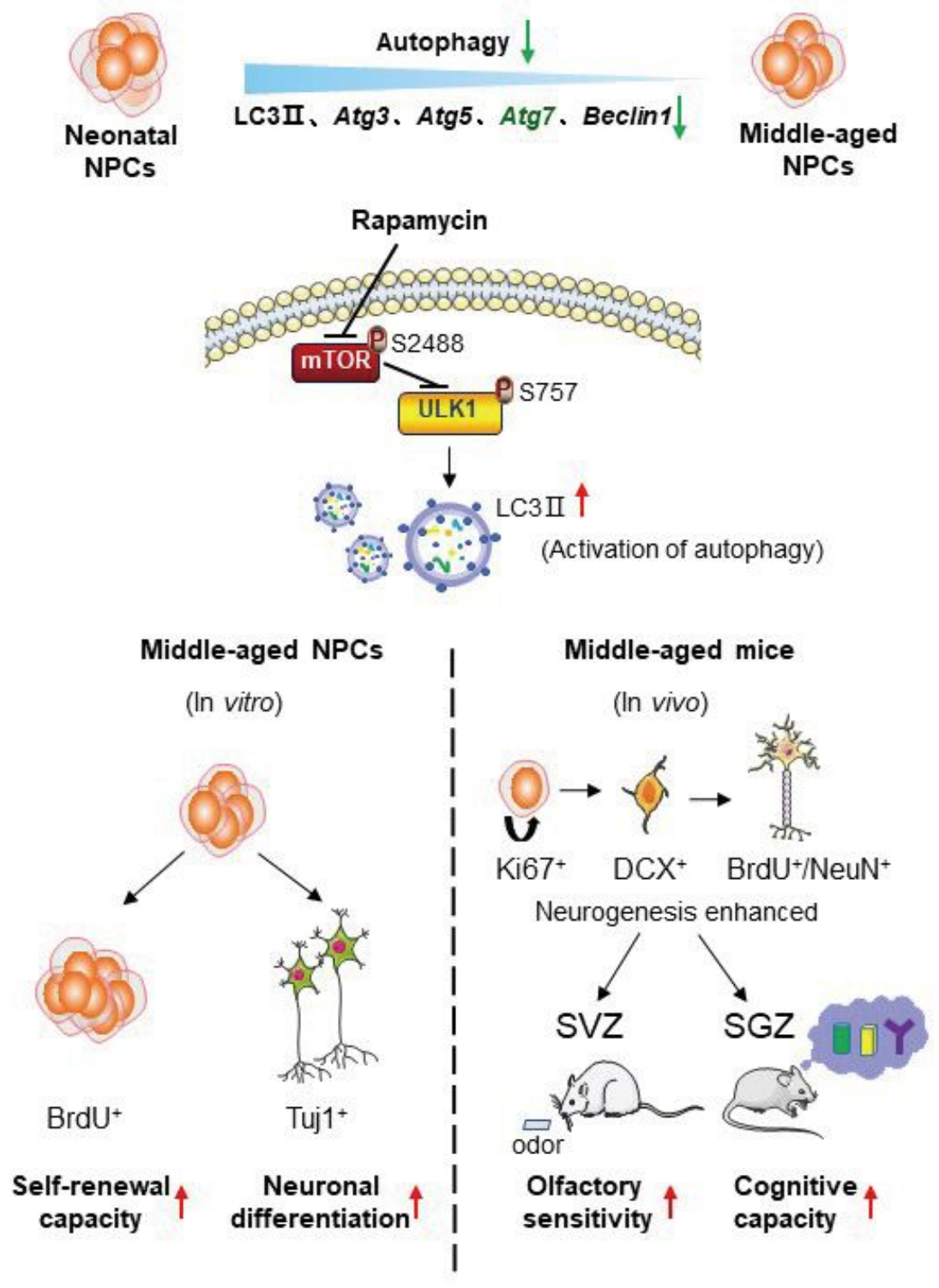

\section{Figure 6}

Schematic illustration summarizing the regulation of autophagy on the adult neurogenesis and neurofunction. The autophagic activity of SVZ/SGZ and NPCs in middle-aged mice significantly declines, compared with that in neonatal ones. Treatment with rapamycin activates autophagy via mTOR suppression, which in vitro revitalizes the viability and neuronal differentiation of middle-aged NPCs, and 
in vivo enhances neurogenesis in middle-aged SVZ/SGZ indicated by improved odor discrimination and cognitional capacities. 